\title{
2
}

\section{Nodes, ways and relations}

\author{
Joe Gerlach
}

\section{Here, now}

Maps, mappings, cartographies; (dis)orientations for the everyday, obdurate disciplinary motifs of and for geography, maligned and admired in variable measure. Cartography; a science and set of practices once pertaining to sovereign power alone, yet now increasingly diffuse in its geographic reach and performance. Nonetheless, whether rendered through hegemonic, quotidian or hybrid assemblages, mapping remains resolutely (geo)political at a range of disparate registers; statist to somatic. Elsewhere, I have used three cartographic attributes, namely those of 'lines', 'contours' and 'legends' as a means of establishing a conceptual vocabulary that underscores the awkward, nonrepresentational aspects of a recent surge in participatory, or 'vernacular' mapping practices themselves propelled by the mass-mediatisation of cartography via mobile, digital and online platforms (see Gerlach, 2014). Here I appropriate a further three attributes; drawn from both the cartographic-lexiconat-large and from the toolkit of OpenStreetMap, the increasingly renowned wiki-based, crowd- and open-sourced mapping organisation established in 2004. These three attributes, nodes, ways and relations, act simultaneously as both the very constitutive material of OpenStreetMap's cartographic output and likewise as points of meditation in this chapter. Together they animate the empirical upshot of the burgeoning conceptual matter on mapping that itself is steadily animating the processual and ontologically/epistemologically insecure natures of contemporary cartography (Kitchin and Dodge, 2007; Crampton, 2010; Elwood and Leszczynski, 2013; Burns, 2014; Caquard, 
2014; Leszczynski, 2014), not least highlighting its radical mutability in time and space (Perkins, 2014).

Centred on a chronologically ambiguous narration of a day's ambulant cartographic-fieldwork with and for OpenStreetMap in the English town of Witham, Essex, the chapter will illustrate how the production of nodes, ways and relations is as much a conjuring of unsettled temporal and spatial sensibilities as it is a charting of 'known-knowns'. In other words, the act of surveying an already well-mapped settlement is far from a redundant act of mimesis, but a geopolitical act of repetition, renegotiation and re-territorialisation of timespaces; the cultivation of what Olafur Eliasson describes as a 'micro-sensibility [of space]' (quoted in Jellis, 2015: 372). Moreover, by thinking about time not as chronological, but as 'durational', after the French philosopher Henri Bergson, it becomes possible to witness the peculiar rhythms of mapping as generative of vernacular and micropolitical space-times. But first, a very brief reprise of contemporary cartography and a word, or so, on vernacular mapping.

\section{Cartography, unbound}

I am in an unfamiliar place ... momentarily what the map tells me bears little sense of where I think I am. (Newling, 2005: 48)

Maps and mappings maintain a curious hold on everyday geographies. On the one hand they enable a slowing down of time-space for navigation, thereby (sometimes) quickening journeys to pre-figured destinations. Moreover, both cartographic reason and the cartographic settlement have helped prise apart a modern constitution of ubiquitous dualisms (Latour, 1993) comprising: rifts between representation and reality, between subject and object, nature and culture, between body and map and indeed between time and space; the geometric logic propagating taxation, territory, terror, war and frontier (Lacoste, 1973). Yet on the other hand, as Newling's bewilderment attests to, maps and mappings continue to disorientate and perplex. The chronologically ritualistic choreographies between bodies and cartographies, between satellite navigation devices and world-weary drivers, for example, manifestly complicates any straightforward division between maps and their users, or between representation and the onflow of reality. At the same time, as enunciated by Nigel Thrift (2012), there is a pandemic underway in mapping technologies and practices, which themselves have recalibrated the natures and geographic character of cartographic performances (Chambers, 2006). This has constituted a move away from a sovereign or statist 
accord of mapping and a shift instead towards a more, broadly speaking, participatory model of cartography, facilitated to a large extent by the recent proliferation in Web 2.0; an 'epoch' of the internet dominated by online interfaces, interactive platforms and protocols (Zook et al., 2004; Zook and Graham, 2007). In calling for a 'cartography unbound', a mapping untethered from the stultifying impulses of representational and temporal certitude, a certain degree of conceptual and empirical disorientation and disruption will be inevitable, such are the vernacular energies required to do the work of unhinging cartography from its determinedly Euclidean grid. The upshot, however, is a re-orientation of sorts; an understanding of cartography that valorises its affective, virtual and performative potential, a speculative mapping that questions not what maps 'reveal', but instead anticipates what kind of worlds and geographies maps could bring into being; this is at the core of the idea of 'vernacular mapping'.

Vernacular mapping acts, conceptually, to accentuate the role of bodies in the folds of experience and to diagram the importance of bodies, human and non-human, affective and virtual, in the distribution and performance of everyday cartographies. It is to accept at all times and spaces that the world is never presented in advance, but that maps and their practitioners are always provisional, subject and vulnerable to ongoing modification and change. The topology of mapping is therefore not the preserve of mental cognitive processes, but a distributed set of bodily, way-finding (dis)orienting performances; a cartography for moving spaces. To speak of vernacular mapping is to shake off the 'geometric habits that reiterate the worlds as a single grid-like surface open to the inscription of theoretical claims or uni-versal designs' (Whatmore, 2002: 6), and is instead to diagram a vibrant cartography that is, "necessarily topological, emphasising the multiplicity of space-times generated in/by the movements and rhythms of heterogeneous association' (Whatmore, 2002: 6).

\section{Stop, momentarily}

‘... everything has its geography, its cartography, its diagram’ (Deleuze, 1995: 33).

\section{Fieldwork traces}

What follows is a fieldwork tracing of a day's mapping with OpenStreetMap in the town of Witham, Essex, UK. As a then newcomer to the OSM venture, part of the motivation was to learn and acquire the skills and literacies required to 'make it' as a competent OpenStreetMapper, while at the same time 
reflecting upon the somatic, affective and micropolitical energies of mapping. Methodologically, this is something of a 'fieldwork of fieldwork' insofar as a central activity of OpenStreetMap is one of 'surveying the field'; a mode of cartographic interpolation. As such, this modest tracing of a day's cartography joins a growing number of studies that approach examples of vernacular mapping in an ethnographic manner (see Perkins and Dodge, 2008); a realisation of human geographers' tentative re-acquaintance with all things cartographic in the face of calls that suggest cartography is facing an existential crisis. ${ }^{1}$

OpenStreetMap is interesting to geographers in three respects. First, OSM is part of a broader culture of crowd-sourcing whereby the production and marshalling of ideas, knowledges, spaces, and indeed politics, is not carried out by an individual entity or defined by a singular subjectivity, but is instead part of an ecology of practices (Stengers, 2005) in which what matters and what is generated is at risk (Latour, 2008), liable to alteration at any moment by any actor, just as cartographically, OSM is itself at risk. Second, despite the relative novelty of OSM's digital platform, there remain strong resonances with the manner in which cartography has been traditionally conceived and practised; specifically, that the role of mapping is to survey, to abstract, to diagram, to render and to contest what is included and omitted from the map, to argue 'what counts'; a performed typology of cartographic orthodoxy that endures its digitisation. Third, there is something to be said about experience and abstraction. OSM deals in abstractions, but it also deals in experiences insofar as these abstractions are generated in the mapping; that is to say that the digital and analogue experiences tied up in OSM cartography amount to vibrant abstractions, further disrupting the Euclidean representational settlement of abstraction held in distinction from reality, divorced from experience; the myth of maps as mirrors of the world (Rorty, 2009).

In this context, by harnessing the experimental tenor of some cultural geographic writings (see, for example Lorimer and Wylie, 2010; McCormack, 2014), an attempt is made to narrate a story that hangs, counter-intuitively, on the disorientation of mapping, but which finds concomitant re-orientations through nodes, ways and relations. It is a story of cartographic bricolage - of making do (de Certeau, 1984); of mapping subjectivities and time-travelling. There is some degree of cartographic orientation on-hand for guidance; these orientations come in the form of GPS traces and geodesic coordinates. The GPS traces are spatio-temporal riffs, the lived, living and to-be-lived traces of walks, bus and train journeys taken in or near the accompanying geodesic coordinates; namely the marker of where the mapping took place. Known colloquially as 'electronic breadcrumbs', they are the spectral remains of cartographic toil, pseudo-memories and digital inscriptions of an event and its lines, but more 
importantly, the knitting together of abstraction and experience; a nod to the interstitial space-times of cartography and the crumbling ontological separation of the world and its representation. Some of the traces also signal failure: GPS calibration malfunctions, random lines generated between errant extraterrestrial satellites and handheld receivers, relations independent of human design, lines, patterns and space-times more broadly emblematic of the setbacks and errors encountered in the performance of vernacular mapping. Similarly, the geodesic markers (longitudinal and latitudinal coordinates) act as spatial refrains, not to anchor the account to any particular locality, but to perform literally as points of departure for the stories, and also to provide resting points for the reader in the same way a map might offer a momentary pause and downward glance from a hiker on the move. Both the GPS traces and geodesic markers are resolutely of a Euclidean vocabulary, but that does not mean necessarily that they must be understood in geometric and cognitive terms alone. These stories, instead, offer a way to think vernacular cartographies through experiential registers.

Witham is a town in Essex, England (Figure 2.1); a reassuringly concrete fact writ-large in the geographic compendium par excellence, the atlas. Witham's cartographic existence is less assured; the rest of it is yet to be mapped, by OSM that is. The GPS handheld device ordered online several days ago has not arrived, and concern is building that I will be turning up to the mapping party on the designated Saturday like a kid arriving at the school gates without uniform. It is bad enough that this will be my first OSM mapping party, previous parties in Maidstone and Haslemere fell by the wayside due to volcanic ash, ${ }^{2}$ but apparently the other mappers did a good job. Not total coverage of the Kent and Surrey towns, but nearly there (as reported by $\mathrm{TimSC}^{3}$ who coordinated the Maidstone mapping party and participated in the Haslemere event). Total coverage? SteveC, founder of OSM, suggested this

\section{LONG. $0^{\circ} 38^{\prime} 11$ " E; LAT. $51^{\circ} 47^{\prime} 60^{\prime \prime} \mathrm{N}$}

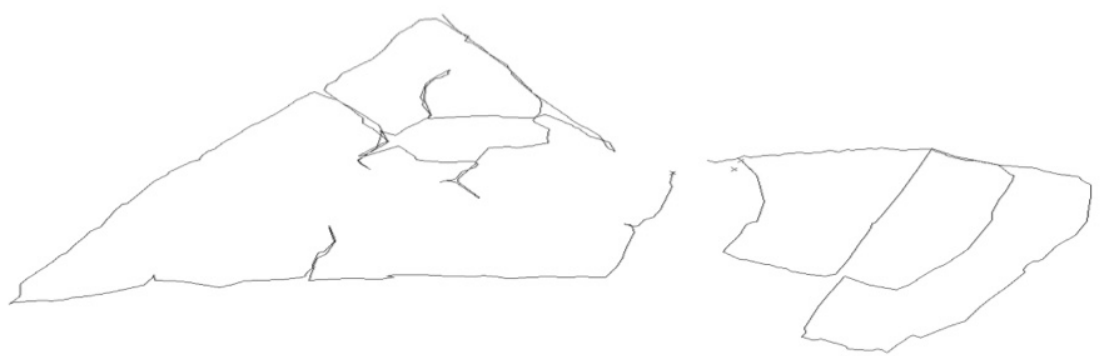

Figure 2.1 GPS route tracing around Witham, Essex (author's image). 
was an ambition OpenStreetMappers needed to divest themselves of, the virtue of a wiki is to always be in the making, never to be complete.

\section{What is a mapping party?}

A big aspect of getting OSM off the ground was the mapping parties: getting drunk and arguing with people. (SteveC)

A mapping party is an event at which OSM newcomers and veteran enthusiasts gather to map an area of the world that is yet to be mapped, or to edit maps already drawn. They are informal affairs held normally over weekends, organised by a clutch of dedicated OpenStreetMappers, invariably book-ended by milky tea in the morning and warm beer in the evening. In the interim, the gathered mappers walk/drive/cycle to blank areas on the OSM map with GPS devices and/or pens/pencils/paper in hand. They set about walking the streets, recording their tracks and marking waypoints and points of interest along the routes taken; all of which are inscribed through either digital or analogue means, or by a hybrid craft of both. Once the points, tracks and lines have been recorded, the data is then uploaded onto one of several OSM map editors and duly edited. The maps are rendered and are, as stated on the website, made available to the world; or at least to those with a broadband internet connection. From the OSM wiki:

The Mapping Party is a convivial, community event. After the mapping is finished, the participants share food and drinks, and enjoy themselves. It's a party, after all! ${ }^{4}$

Why Witham?

Again, from the wiki:

Although a residential land-use area has been added for Witham, very few of the roads have yet been added. Checking the 'nonames' layer also shows that most of the roads currently mapped are lacking street names. As an introduction to OSM, Witham therefore lends itself to both GPS mapping to add missing roads and paths, and walking papers to add missing road names and Points of Interest (POI). It is believed that there is also a shared use cycle/foot path running parallel to an ever increasing length of the A12 which is currently unmapped. Some of this might also be mappable. ${ }^{5}$

A fieldwork scrapbook (Figure 2.2):

It is an early start on a Saturday morning, travelling from Oxford to Witham via London. Changing trains at Didcot, boarding Coach D. Peering down the 


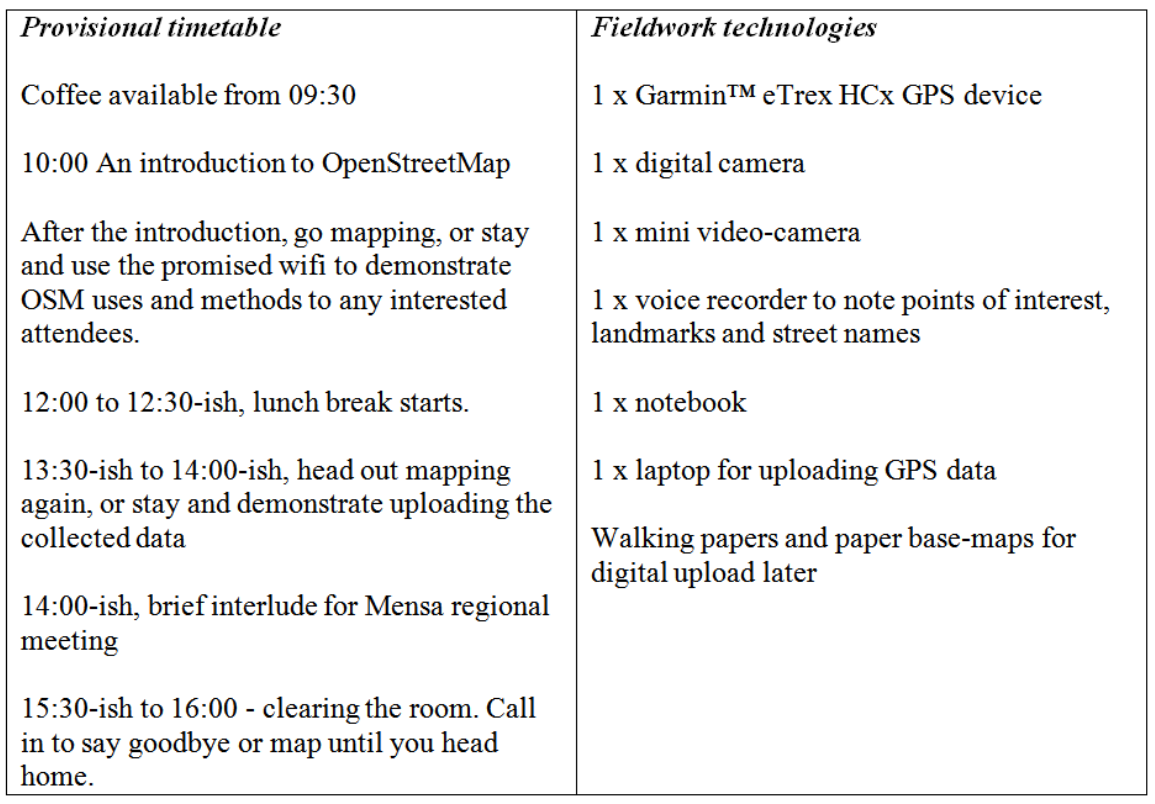

Figure 2.2 Fieldwork scrapbook extract from author participation in OSM mapping party (author's image).

STOP PRESS: The GPS device has just arrived in the post. Excellent news.

LONG. - $1^{\circ} 15^{\prime} 59^{\prime \prime} \mathrm{W}$; LAT. $51^{\circ} 44^{\prime} 53^{\prime \prime} \mathrm{N}$, heading south-easterly

Figure 2.3 GPS route tracing author's movement south-easterly while mapping Witham (author's image).

carriage to see a dozen or so early risers staring at newly installed television screens on the backs of seats. No one has paid the $£ 3.95$ subscription to watch re-runs of trash television. Instead everyone is making do with watching a yellow spot flashing in the centre of a map. As the train pulls out of the station (speed: 16, 17 ... 18mph; altitude: 177ft), the flashing yellow spot remains fixed in the centre, but the base map slips from right to left at jagged intervals; from Didcot, down a touch to the south-east, and on to Reading (figure 2.3). In this instance, CloudMade, a company founded by OSM enthusiasts and now sponsoring OSM technically and financially, has used the base map generated by OSM (the map created and edited by OSM users) and customised it for the needs of the train company; superimposing a separate GPS-controlled 


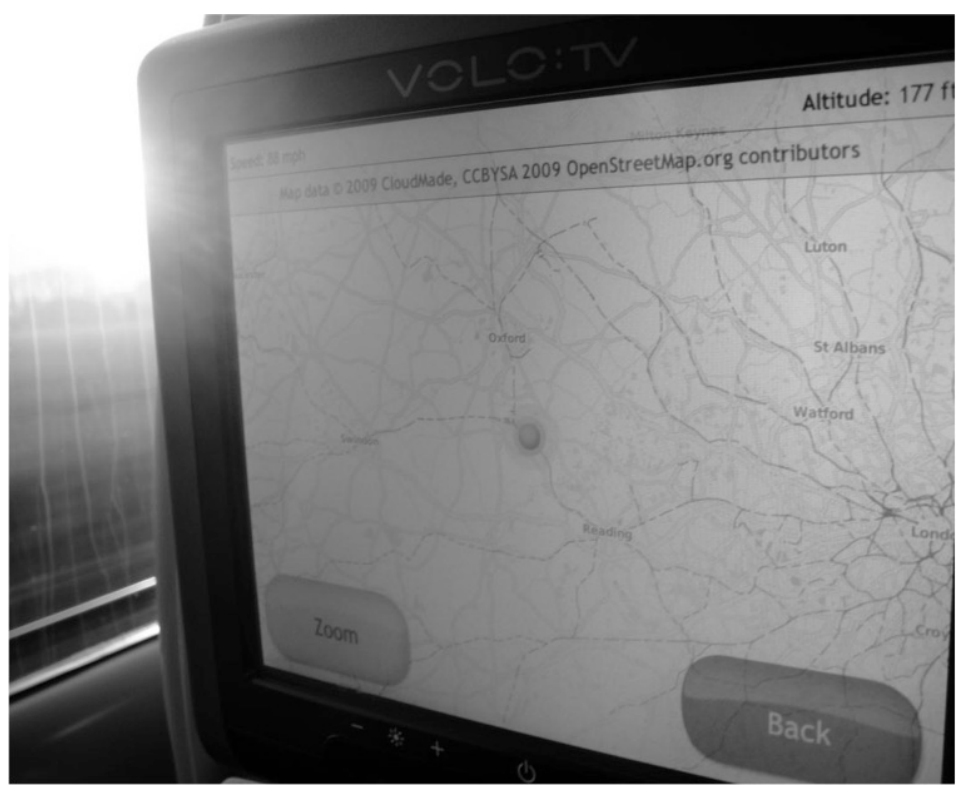

Figure 2.4 Author's train journey photograph demonstrating the train operator's use of OSM base maps in passenger navigation screens (author's image).

cursor upon the base map to geolocate the train for the duration of the journey (Figure 2.4); a good example of a mapping-mashup using copyright restriction free data. Sitting down, rushing all too earnestly to get the map displayed on the individual back-of-seat screen; surprise and smugness on realising that the map had been generated with OSM data. Serendipity; the anorak, vernacular, geeky subjectivities of train-spotting and the lines, contours and legends mapping collide in Coach D. Crowd-source mapping was making its mark in public.

The emerging map plays into the (un)folding of both the journey's duration and the modulation of the carriage's muted affective atmospheres (Bissell, 2010). But the earlier sensation of smug amusement is an isolated one; Bergson provides the cold water:

it may, perchance, have happened to you, when seated in a railway carriage ... to hear travellers relating to one another stories which must have been comic to them, for they laughed heartily. Had you been one of their company, you would have laughed like them; but, as you were not, you had no desire whatever to do so. (Bergson, 2008: 11)

Onwards. 


\section{LONG. $0^{\circ} 38^{\prime} 11 " \mathrm{E}$; LAT. $51^{\circ} 47^{\prime} 55^{\prime \prime} \mathrm{N}$}

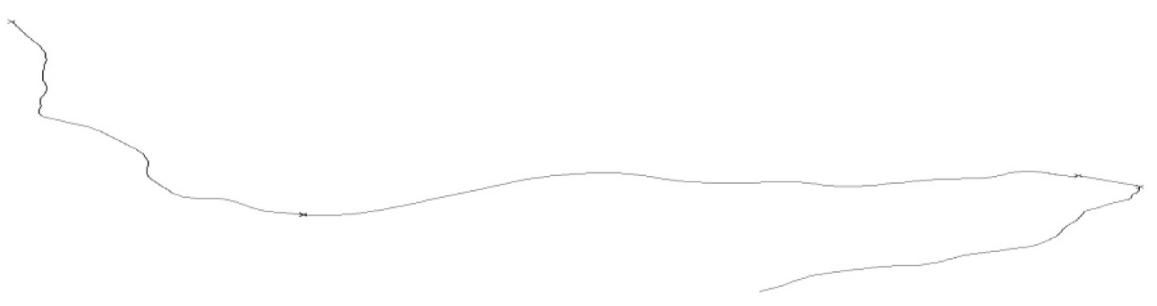

Figure 2.5 Author GPS track (author's image).

'I don't think we've met before', said Ed. No we hadn't, but he interrupted gluing posters in the window panes of the White Hart Hotel to shake hands. Ed had organised the Witham mapping party and was setting up his PowerPoint presentation that, for today only, had a dual audience as the event was timed to coincide with a regional meeting of Mensa (of which Ed is regional secretary). 'You do know there is a Mensa meeting on later this afternoon?' he asked. Yes, I knew, even if the IQ was not up to the job. Ed is from Clacton-on-Sea, not far from Witham, and is proud of his role in mapping most of Essex from Billericay to Walton-on-Naze for OSM. How did Ed get involved? His friends had been doing some mapping, so he brought himself a Navi ${ }^{\mathrm{TM}}$ GPS device and set off on his bike through the country lanes surrounding Clacton. It appealed to his professional sensibilities as a software programmer. An Oxford educated mathematician, Ed is keen on detail, and prefers accuracy to guesswork.

The previously quiet function room, carpeted in 1970s decor suddenly reverberates with the strange beats of something akin to elevator music on steroids. Ed had loaded a video called 2008: A Year of Edits. Produced by ITO World, a data visualisation supplier which, in common with CloudMade, has a quasisponsoring relationship with OSM, the video shows a spinning globe set against the pulsating sound track as lines flash across parts of the planet, each flash animating a newly generated addition or edit to the OSM map during 2008. The brightest and most intense flashes occur where one might expect them to occur, in Europe and North America, but there are not many places left on the planet where the odd trace has not yet permeated. India strikes me as particularly bright. Flashing nodes of light are backed by the steady pulse of a synthesised bass drum; each pulse proclaiming OSM's mark, each beat beckoning for more to come; an expectant, anticipatory rhythm of simultaneous cartographic past-ness and futurity.

The beat ongoing, we discuss the recent efforts of OSM in mapping Haiti following the massive earthquake that struck the country in January 2010. Ed did a lot of mapping of south-west Haiti, deliberately avoiding Port-au-Prince; 
the area that had received most of the mappers' attention and cartographic efforts. The difference in OSM coverage between the pre- and post-earthquake maps is stark; more so because these maps had been rendered without taking a single step in Haiti itself, but traced remotely from CIA and Yahoo satellite imagery.

Pause, for one second; perhaps that is not entirely true. We look at the 'raw' data base layer of Haiti; there are a cluster of thin blue lines in the corner; blue lines denote user-generated GPS traces, which in turn suggests that there are OSM mappers actually present in body in Haiti. I'm surprised, Ed is taken aback. Until, that is, we zoom out and realise we are not looking at Haiti at all; we are gazing at a base layer image of Clacton, Essex. Awkward, but demonstrative of a vernacular myopia when everything is reduced to its cartographic data primitives such as nodes, ways and relations. Clacton and Port-au-Prince do not appear that dissimilar; a pointer towards the familiar limits of abstraction from the world and the assumptions made about those abstractions. When the world is packed into lines as it is in this case, it serves as a reminder of how pervasive cartographic reason has been in Western thought and governance (and by extension, in everyday life); that disparate assemblages, cultures and things can be convened and disciplined through geometric abstraction.

OSM user 'Nigel' sends a text to Ed, he can't make it today, he is going to the theatre in Yorkshire tonight; a long drive ahead. Participation at such events is always precarious.

Ed was preparing an introductory talk on mapping techniques and editing software for his Mensa colleagues and other interested mappers, of which there were not that many, fourteen of us in total, if that. He outlines OSM's reasons for being, and the utility of the map; it can be crafted to any use the user wants, whether it be for cycle maps, maritime charts or, apparently, icing cakes. Cakes are in fact integral to OSM lore for two reasons. First, the areas to be mapped are transformed into 'cakes', or rather diagrams that are used to divide the area of concern into identifiable, digestible tracts; a cartographic mapping tool borrowed from baking to order, discipline and manage who maps what. Second, cakes form an important litmus test of whether a map is genuinely free from licensing restriction. For example, to ice a cake with the transfer of a map sourced from Google would be to violate Google Maps' Terms of Service (which states that derivative works, even iced cakes, are not permissible). Conversely, to ice a cake with a transfer of an OSM map is entirely acceptable, assuming that the resulting cake is available to share, in both its derivative forms and its devouring. It is in these micropolitical (and simultaneously macropolitical) gestures that OSM and similar groups stand out from their traditional cartographic antecedents; characterised by a stubborn refusal to 
be reined in by copyright restrictions or corporate vindictiveness, and instead marked by an editing of the world that produces other forms of knowing and navigating space, whereby the map is not mimetic, but instead a device, a tool, a technique, a cake for being in the world.

2008: A Year of Edits is stuck on a continuous loop; grating, dislodging timespaces to think. Mensa members interject with questions; 'what scale do you render the maps?' 'Isn't that data copyrighted?' 'If we mark a waypoint in front of a pub, then we'll be marking where our bodies are located in front of the pub, not marking the pub itself; how can we be accurate?' 'What projection are we using here... a transverse Mercator, yes?' A transverse Mercator? ${ }^{6}$ Even Ed was stumped. Data copyright? This is an awkward one. In April 2010, Ordnance Survey was forced into releasing a vast amount of geo-data that had previously been under strict licensing and commercial control. Not anymore; now anyone can access the Ordnance Survey 'Open Data Service', which includes street map data. Consequently, to reproduce this data without permission is now entirely legal. A great success surely; the outcome OSM had been fighting for ever since its inception? Technically yes, but Ed looks a little forlorn. Now that OSM users can use Ordnance Survey maps as base-level, third-party imagery, he was concerned that no one would turn up to Witham. Why get up and walk around Witham when one can sit at home and transpose data from one map to the other? Indeed, why bother at all? All the questions that emerged digitally in the mail-lists previously now find themselves posed in analogue.

Tellingly, OSM urge users to cultivate experiences in an empirical and bodily sense, to allow the 'field' to underpin and legitimate the maps generated. Experience being understood here as, 'the instant field of the present [that] is always experienced in its “pure” state, plain unqualified actuality' (James, 2003: 69); experience as the cornerstone of vernacular activity. Paul Carter asks the question, how can we live with our maps differently, away from an understanding of maps that renders them as hegemonic or disempowering (Carter, 2009)? One response might be that editing the world with OSM offers a way of living differently with our maps through the valorisation and pursuit of experiential cartographies that involve messy movement traces, 'a geo-graphy, where geography merges into performances to produce a different design in the surface' (Carter, 2009: 14); a vernacular mapping.

Bodily experiences too are central to the performances of being an accomplished amateur as Hennion (2007: 101) remarks, 'the meticulous activity of amateurs is a machinery to bring forth through contact and feel differences infinitely multiplying'. However, simply because OSM relies on the work of amateurs themselves working through and generating vernacular sensibilities, 
it does not follow therefore that some form of disciplining is absent from the practice of vernacular mapping. On the contrary, these vernacular impulses are trained by informal hierarchies of experience, technologies and conventions that promote a regime, or at least, a sensibility of self-governance and selfdiscipline. For example, in relation to disciplining the amateur, cartographic 'errors' present in the map are detected by automatic scripts, algorithms and programme robots (created by OpenStreetMappers who possess programming skills). On detection, errors are deleted automatically, a record of which is posted publically on the OSM wiki; a list-like discipline of name-and-shame that quickly striates the amateur-amateur from the amateur-expert, and to follow Schaffer (1988: 119) in relation to amateurs and astronomy, "mere" observers [become] relegated to the base of a hierarchy of management and vigilance, inspected by their superiors with as much concern as ... the stars themselves'.

Mapping: 'It takes close attention to see what is happening in front of you. It takes work, pious effort, to see what you are looking at'. (DeLillo, 2010: 16)

What might count as the cartographic node of vernacular or micropolitical sensibility here is the manner in which the OSM mappers attend to their matters of concern in a 'perplexed' mode. To paraphrase Hennion once again, for OSM mappers to work in perplexed mode means to be on the lookout for what mapping does to them, to learn to be affected, to be 'attentive to traces of what [mapping] does to others; a sharing out among the direct sensations to be experienced (or whose experience is being sought), and the indirect relays that permit one to change one's own judgement a bit, while relying in part on the advice of others' (Hennion, 2007: 104). OSM could be said to rely upon these embodied experiences as central to its propagation and maintenance because it is in these experiences that a particular sociability and micropolitical sociability is cultivated; sensibilities that sustain and promote a variegated, creative and sometimes baffling expertise; the hallmark of OSM's cartographic energies. The cartographer does not precede the mapping. Instead the cartographer and the mapping are co-constitutive, always in the making.

Circle back to the event. Witham, still.

Ed has finished his presentation, and spare GPS devices are loaned to some of the other first-time mappers. Everyone starts pushing buttons. Some walk out of the pub straight away, they know what they are doing. They are OSM veterans. For others, looks of extreme bewilderment become etched on their faces. It is going to be a long morning. 'Don't worry', says Ed. 'OSM is just about nodes, ways and relations'. 
Stop there. Nodes, ways, relations?

Node: A node is the basic element, building block, of the OSM scheme. Nodes consist of latitude and longitude (a single geospatial point).

Way: A way is an ordered interconnection of at least two and at most two-thousand nodes that describe a linear feature such as a street, footpath, railway line, river, fence, power line, area or building outline. One way is characterised with uniform properties; for example, priority (motorway, trunk roads), surface quality, speed, etcetera.

Relation: A relation is a group of zero or more primitives (nodes and ways) with associated roles. It is used for specifying relationships between objects, and may also model an abstract object.

Nodes, ways, relations; the OSM data 'primitives', the cartographic building blocks of the OSM map, but taken further they are also constitutive of a geometry of temporalities and subjectivities in the making. If nodes, ways and relations are the digital protocols of how the map can be drawn and edited, then so too are they the analogue and affective functions of a vernacular subjectivity; cartographers as nodes, as individuated actants, but nodes only insofar as they are put into motion with other mappers and technologies to generate ways and relations; ways as technologies bringing cartographies into being, and relations as the necessary co-fabrication of both the maps themselves, but also the space-times and events in which the map is forged; "the relation itself is a part of pure experience; one if its "terms" becomes the subject or bearer of the knowledge, the knower, the other becomes the object unknown' (James, 2003: 4). Micropolitical, vernacular subjectivities are thoroughly relational; co-constitutive relations and assemblages of technicity, expert and amateur bodies. Such subjectivities promote creativity and expertise outside of and beyond proscribed institutional norms. Much like the movement embedded in maps, to paraphrase Carter, this geometry of subjectivities is neither fixed nor linear, but instead rhythmic; a pulsating geometry of relations and creativity through which maps and micropolitical gestures emanate (Carter, 2009). Nodes, ways and relations, then, are more than editorial metaphors for the assemblages at work here, instead they are more urgently caught up in the very cultivation of these cartographic practices and spatio-temporal assemblages.

Nodes, ways, relations, then.

Still some looks of bewilderment and the pace of the day thus far has been sluggish at best. Time to inject some impetus. 'Let's just get out there', suggests Ed; good idea. First, we need to cut the cake, or more specifically, the sector cake of Witham; the town divided into palatable areal chunks that lack roads, points of interest, or are indeed devoid of mapped space altogether. We pick 
LONG. $0^{\circ} 38^{\prime} 11^{\prime \prime}$ E; LAT. $51^{\circ} 47^{\prime} 52^{\prime \prime} \mathrm{N}$

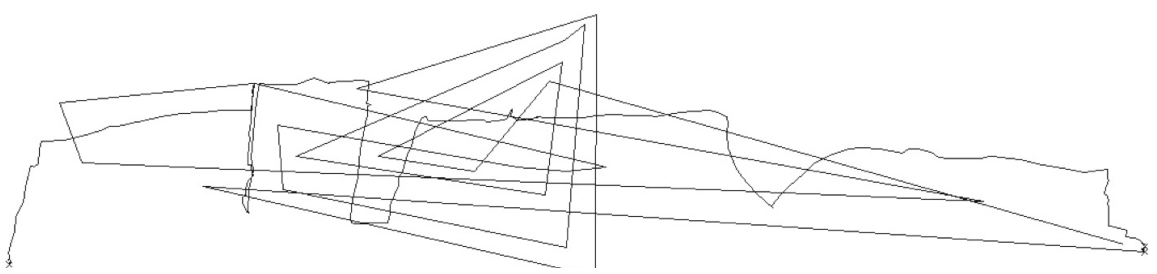

Figure 2.6 Author GPS track (author's image).

the slices of Witham that we want to traverse and map (see Figure 2.6). Some pick slices nearby the pub, some pick slices that involve a riverside walk. I pick slice eighteen. No more than a lucky number, a gut feeling, and something alluringly trapezoidal about the slice. Ed passes me a walking paper for slice eighteen on which he has already kindly outlined the sector with an orange highlighter. Sipping quickly the last dregs of coffee, we say farewell for now. Slice eighteen is ten minutes' walk south of the pub.

\section{Acquiring satellites}

So this is it. Isolation.

That, as ever, is not strictly true; the GPS, the voice recorder, the walking paper, the camera, the fieldwork mentality; faithful companions all the way.

On reaching the edge of slice eighteen I am immediately lost. The edge of the cake starts on Laurence Avenue, but I'm stood on Howbridge Road. Looking at the walking paper, and other maps of Witham I had printed the day before, an irony grows; that to make a map is to need a map of the area you want to map. Standing under the nearest bus stop for shade, searching desperately for Howbridge Road to transform into Laurence Avenue, but the map is having none of it. Orwell's (1938: 58) Spanish civil war encounter with a naive militiaman in Catalonia springs to mind, 'obviously he could not make head or tail of the map; obviously he regarded map-reading as a stupendous intellectual feat'.

The mapping stops before it even begins.

'What good is time?', Henri Bergson (1992: 93) asks mischievously. Not much good, if we are to take time to be a matter of chronological measure. Consider, for example, how the history of cartography is problematic insofar as it can be told as a linear, hubristic tale of modernisation and progress. Vernacular mapping is not reliant upon such metrics of the clock and the timetable. It has no time for such time. Bergson provides us with another formulation of and 
for time, premised on the metaphysical acceptance of indivisibility and the reality of constant change; 'we shall think of all change, all movement, as being absolutely indivisible' (Bergson, 1992: 142). This, unsurprisingly, extends to Bergson's figuring of time as 'duration'. While Bergson admits that there is 'succession' in the movement of time, he refuses the neat compartmentalisation of durational time into chronological units. To be adumbrating about duration, it is to state that past, present and future are constitutive of one another - they enfold, they are indeed, indivisible; duration cannot be measured, it can only be intuited, and, as Deleuze (2004: 33) proclaims, 'intuition is the joy of difference'. Perhaps in the annals of cartography, a mapper's reliance on sensibility, intuition and virtuality is underplayed at the expense of realising the unabated difference that maps can produce. And don't think of intuition, intuitively; to intuit takes skill and requires a kind of intellectual and somatic empathy with the matter that one is interested in; an 'intellectual sympathy by which one places oneself within an object in order to coincide with what is unique in it and consequently inexpressible' (Bergson, 1992: 23). That duration is not chronologically metered does not mean that there are no contours, disruptions or undulations in duration; crucially, duration still permits an acknowledgement of rhythm in producing space and life itself.

Mapping is replete with concatenated rhythms that call upon the durational movement of pastness, presentness and futurity. Take, for example, the following encounter between Earth and its immediate orbital space.

Lost, still. Turn on the GPS device, this will help for sure. 'Acquiring satellites' reads across the GPS screen. 'Acquiring satellites' - when did that become an everyday matter? Such acquisitions are now minor moments of geopolitical connection that have become a necessary step for locating position in the absence of accurate paper maps, local knowledge and common sense. After two long minutes, the GPS has a three-dimensional lock on my position, meaning it can detect both altitude and geodesic position. Cosmic numbers to be sure. Back down to Earth, quickly, though suffusing the duration of mapping is a continuous spatio-temporal 'lag'. A signal's journey between satellite and handheld device might appear quick from a cognitive, or even straightforwardly chronological, perspective, but there is still a delay as signals negotiate with nonhuman interfaces before travelling the leagues between Earth and orbital space. This spatio-temporal lag is liable to create a 'slowing down' of events, but from a durational perspective, it protends the present (extending a present-ness, or 'now' by stretching the past and future). Thus, as the signals reach the handheld device from a contracted 'past', the waiting, anxiety and anticipation for signal recognition opens up a virtual space-time in which mappers can learn to map differently, or to be 'creative' in the way they manage that lag. Lag, insofar as it 
is evidence of a durational, temporal 'stretch', necessitates creative, vernacular responses.

The lag extends/protends, and disorientation grows.

A road name would do for now, but even that is not forthcoming, as the GPS is only pre-loaded with a perfunctory road map of the world. A further $£ 150$ would buy a premium upload for UK road data (Ed later tells me that I should have downloaded OSM base maps on to the GPS for free. I'll do that when I get home). The GPS is not helping, so in walking a little further to the next street, I realise that I am in the right place, but that the OSM walking paper is inaccurate; not entirely unexpected, as this was one of the very reasons for convening a mapping party in the first place. My first edit; delete Laurence Avenue, replace with Howbridge Road; just scribbles on the walking paper. Stood on the corner of Pelly Avenue; this is where I'll make a start. It does not yet exist on the OSM map, so for all concerned, it is somewhat new territory. There was no such novelty for the passer-by who asked if I was lost; 'are you ok, you look lost?'. Spot on. I have no idea where I am, but I explain what I'm doing, and thank them for their help. They walk off, nonplussed. The GPS device is back on; holding down firmly the cursor button on the GPS to mark a waypoint. Point 001. Over the course of the day, I mark seventy-two waypoints, designating street corners, points of interest, and anything else that struck me, literally on occasion, along the way.

Mapping, vernacular or otherwise, demands multi-tasking; sketching an outline on the walking paper as one goes, maintaining orientation, marking waypoints, checking the status of the GPS, labelling all points of interest (including postboxes), taking photographs, making voice recordings, ensuring to walk down every road, every pathway, every cycle lane, making sure that the trajectory of one's walking route is not too erratic so as to generate a bizarrely stochastic electronic breadcrumb trail on the GPS which would become a nightmare to upload and render into a map; yes, the rhythm can be as breathless as it reads. While the GPS takes care of the lines walked, I struggle to mark points of interest in-between the lines, approximating their position on the paper map. At the same time, I and the other mappers (wherever they have gone) need to appear inconspicuous and non-intrusive. Understandably a few glares from residents emerged, watching my cyclical and repetitious movements around the estate and forced at one point into a double pirouette to calibrate the GPS compass. Irksome, I already had a sense of where north was without the dance, but at least now we're calibrated. More importantly, the GPS acts as an authenticator of presence, a reliable witness in the mapping; it feels that to be without one would be to jeopardise the sense of cartographic legitimacy OSM is trying to engender. 
Walking with the head down, peering at the traces emerging on the GPS, pacing at around 3.7 miles per hour (is that slow?), but there is no sense of scale, so pencil lines are crammed, depicting roads into an unnecessarily small sketch map, not entirely confident that this is not a mistake, or that experienced OSM users won't make derisory comments about the day's mapping when GPS traces are uploaded on to the OSM server. The frenetic rhythms of charting, anticipating and walking have a disparate effect on cartographic attention. On the one hand, the stuttering rhythms provoke simple lapses in concentration; what was that street called, where have I just been? On the other hand, the durational quality of the mapping conversely focuses a different kind of affective attention to the virtual; the on-the-cusp anticipation of what's next, where am I headed? To this end, and from an instrumental perspective, one might argue that given the idea of cartographic duration, neither a slowing down or speeding up of the mapping's rhythm would necessarily enhance or worsen the quality of the cartography. Back to space, and from a God's trick perspective, the number eighteen slice doesn't appear that large. But of course walking in and out of its labyrinthine roads and pathways soon elucidates the feeling of considerable areal space, and a realisation that a cartographer will never capture everything that is here, even in a lifetime of mapping. This was going to take several hours, so I ring Ed and tell him I'm skipping lunch to continue mapping (it extricates me from the Mensa meeting too).

Relations with the GPS device are ambivalent. On the one hand, their helpful triangulation of position can provide moments of endorsement; guarantors of location. 'You are here'; you are in the right place; human and non-human in geometric harmony. On the other hand, they stir tension, argument and tears, setting bodies off on labyrinthine nightmares, to the edge of their worlds. Safety net or banana skin, GPS devices have replaced protractors and compasses as the cartographer's instrument of choice; powerful remote-sensors that have revolutionised mapping procedures, but devices that have something of the Latourian black-box about them. As suggested earlier, calibration failures, glitches, and even lags, far from being setbacks, are generative points of contention, talking points for cartographic hobbyists; problems that require solutions. In sum, despite OSM's strive for accuracy, the ethos of failure is one that is cultivated and celebrated as a productive ethic in experimentation; if you have an idea, or a glitch, do not, as OSM's founder suggests, worry. Instead, 'JFFI: Just F* cking Fix It' (Coast, 2010: no pagination). This is, after all, vernacular.

Continue walking, continue mapping slice eighteen. A Witham's summer day, the neck starting to feel the sun's presence more urgently; a somatic, durational beckoning for the day to be done - soon. There's Howbridge Junior School, there's the Jack and Jenny Pub, there's the bus stop for service ninety. 
LONG. $0^{\circ} 38^{\prime} 11^{\prime \prime}$ E; LAT. $51^{\circ} 47^{\prime} 52^{\prime \prime} \mathrm{N}$

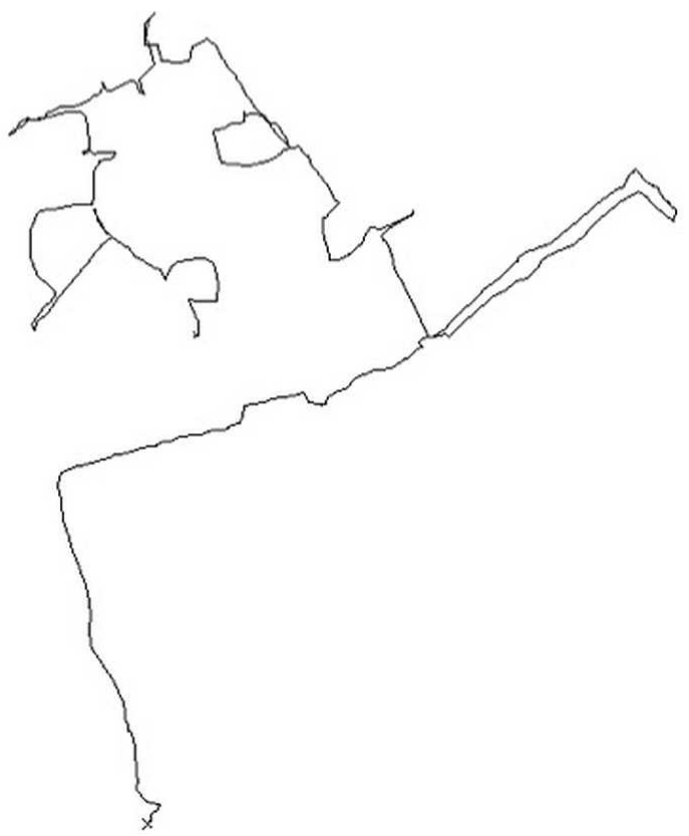

Figure 2.7 Author GPS track (author's image).

All waypoints, all useful nodes of information; mining for data, gorging on GPS signals; guilty pleasures for sure. Tongue in cheek, this feels like a civic service, a good deed in the mapping, though this kind of vernacular mapping shouldn't be beholden to such stilted, do-gooder politics. Slice eighteen has a rhizomatic quality about it; there is a road through the gap in the houses, but I don't know how to get there, and I cannot be sure that the path has not already been walked. Two, slightly off-parallel thick lines on the GPS suggest that indeed I've been here before, so doubling back on myself, a pathway missed has been found. Kids on bikes ask me what is going on, so I ask for their help; which streets do they live on, what cycle tracks do they use? It is a brief but useful vernacular cartographic event, a mini-convening of publics; a distributed kind of wayfinding with others and a type of conversational mapping which has a heritage far longer than paper bound, Euclidean cartography.

In duration, four hours pass extremely quickly, but in a spirit of cautiousness, I loop around slice eighteen once more, treble-checking that the GPS traces have been saved to the memory card, with waypoints intact. Time to head back to the pub. A few other OSM users had turned up who had not been in attendance in the morning. Most of them drove their cars around the bypass and environs 
of Witham, collecting vast amounts of geo-data from just a few hours work. Our function room booking is about to expire, chronological time is against us, so Ed and the others provide a rapid tutorial on uploading the data to the OSM server and then using various OSM editing packages to render the map. One last question before we depart: 'Do you find OSM mapping addictive?', I ask the assembled mappers. 'Addictive is probably the wrong adjective to use', replies Ed, offering no alternative. This is the problem when academics attempt to impose the categorical upon the non-categorical. So what is this vernacular mapping? Perhaps it is an obsessive compulsion in the mapping, the quest for spatial affirmation and a sheer junketing for the ludic qualities of this kind of mapping (Perkins, 2009); perhaps it is an experiential jouissance to mapping not found in the state-led cartographies of early nation-state Europe. Maybe vernacular mapping, and part of its contemporary appeal, is about the opportunities for time-travel, in durational sense. It could be all, or none of the above. In one sense, the likes of OSM dos not mark reclamation of mapping for the everyday, as people are mapping all the time in some form or other, but what it does do is disturb and inflect the meanings of Euclidean cartography; where what counts as accurate and precise is up for grabs.

\section{Coda}

I tried not to think beyond geography. (DeLillo, 2010: 101)

Those urges to capture everything, to mark nodes, ways and relations, to monopolise movement cartographically, to laud diagrammatically over Witham as Captain James Cook did so in Botany Bay, Australia (Carter, 1987); where do these urges come from? In part, they stem from the discursive and cultural baggage that has become welded to cartography; the discipline's associations with colonialism, monopoly and meta-narrative. Importantly, the focus of this chapter on the experiential is not meant as a disavowal of other well-known linguistic and discursive registers of cartographic practice. Following Wylie (2002), to divorce the experiential from the discursive in its entirety would be somewhat disingenuous. In the case of pacing around Witham, swashes of Cartesian and Euclidean thinking pervade the somatic rhythms of mapping, as bodies are simultaneously orientated and disorientated by cartographic norms and orthodoxies that have been codified and handed down through generations, through textbooks and now through wikis.

That does not then entail a valorisation of Euclidean cartography or Cartesian logic, it is merely a way of writing-otherwise these mappings, of thinking 
through how notions such as objectivity and drawings such as straight lines, while seemingly disciplinary and stifling, can actually be generative of a panoply of bodily dispositions, and micropolitical possibilities. Put simply, to achieve and trace a straight line for OSM in Witham, bodies must move, sweat, ache, meander and blister. They need to coalesce with non-human bodies and learn to be affected in the process. It is then the virtual potential of these cartographic bodies in motion that invigorates these kinds of cartographies, whereby their production and affects are recombinant and continually at risk, not pre-figured as per the cartographies of past officialdom.

What of the time travelling engendered by these moments of participatory cartography? This requires a return, obliquely, to Henri Bergson's (1992) thinking on time. If, as Bergson implores, time is figured qualitatively as 'duration', rather than as 'chronological', then the former makes indivisible the fractures between past, present and future and transforms, instead, their matter to exist on the same plane of immanence. To read time by the latter, chronologically, for Bergson, is to spatialise time - to crystallise spatial units of time into the hours, the minutes, and the seconds that flow by. In other words, Bergson is castigating a cartographic figuring of time and so on this claim alone, a map would be understood as freeze-frame of a particular time. Yet, not only is Bergson's rendering of space ironically reductive, but participatory mapping also helps to illuminate the indivisibility of time and space, and indeed to demonstrate the durational qualities of space (and not just of time). Mapping here is not about creating spatially or temporally bound segments of experience in order to create similarly segmental abstractions. Instead, nodes, ways and relations are interstitial points of departure; or to borrow from James Ash (2015), they might be figured as 'envelopes' of space-time; foldings that call on the past, that beckon a futurity while simultaneously propagating a cartographic present. The temporally awkward narration of these mapping vignettes points to the indeterminacy of the future, even if much of the mapping is seemingly mimetic, based upon already well-annotated streets and pre-figured way points. This might not be time-travel in a register figured by H. G. Wells, for, as Elizabeth Grosz (2005: 10) states, 'durational force, the force of temporality is the movement of complication, dispersion or difference that makes any becoming possible and the world a site of endless and unchartable becomings'. Quite so. Why fix the world cartographically in the chronological past, lest difference be numbed? Nonetheless, I would argue that becomings are 'chartable', but only if one charges that charting, or that cartography with a vernacular ethos which itself hinges on never-quite-knowing; of finding joy in intuition and wayfinding through spatial and temporal disorientation. Cartographic temporality then, in a vernacular sense, and as exemplified to a degree by 
OSM, might be understood as a tentative, speculative time-travel; a mapping not based on the certitude of chronology, but through the spatial-temporal indivisibility of duration.

\section{Notes}

1 Boria (2013) also notes the increasingly strained relations between geographers and cartographers.

2 The 2010 eruptions of the Eyjafjallajökull volcano in Iceland caused widespread disruption to aviation across north-west Europe for several weeks. The matter of concern or manifested controversy here, however, was less the particles of volcanic ash themselves, but their cartographic rendering and modelling. Competing measurements of aircraft tolerance to differing levels of ash by multiple jurisdictions highlighted the awkward, mischievous and politically febrile entanglements of scientific representations (see Ulfarsson and Unger, 2011).

3 OpenStreetMappers are referred to by their chosen user names, e.g. TimSC and SteveC.

4 Mapping parties. [Online] Available at: http://wiki.openstreetmap.org/wiki/ Mapping_parties (accessed 23 November 2017).

5 Witham/201004 Mapping Party. [Online] Available at: http://wiki.openstreetmap. org/wiki/Witham/201004_Mapping_Party (accessed 23 November 2017).

6 Developed protractedly by Carl Gauss and Johann Krüger in 1825 and 1912 respectively, the Transverse Mercator projection retains the central meridian line of the original Mercator projection, but unfolds the image of the Earth in a concertina fashion so as to display the terrestrial poles (Jackson, 1978) and narrow the proportional areal disparities between landmasses.

\section{References}

Ash, J. (2015) The Interface Envelope: Gaming, Technology, Power. London: Bloomsbury.

Bergson, H. (1992) The Creative Mind: An Introduction to Metaphysics. New York: Carol Publishing.

Bergson, H. (2008) Laughter: An Essay on the Meaning of the Comic. Rockville, Maryland: Arc Manor.

Bissell, D. (2010) Passenger mobilities: Affective atmospheres and the sociality of public transport. Environment and Planning D: Society and Space, 28: pp. 270-289.

Boria, E. (2013) Geographers and maps: A relationship in crisis. L'Espace Politique, 21. [Online] Available at: http://espacepolitique.revues.org/2802 (accessed 3 August 2016).

Burns, R. (2014) Moments of closure in the knowledge politics of digital humanitarianism. Geoforum, 53: pp. 51-62. 
Caquard, S. (2014) Cartography II: Collective cartographies in the social media era. Progress in Human Geography, 38: pp. 141-150.

Carter, P. (1987) The Road to Botany Bay: An Exploration of Landscape and History. Minneapolis, Minnesota: University of Minnesota Press.

Carter, P. (2009) Dark Writing: Geography, Performance, Design. Honolulu: University of Hawaii Press.

Chambers, R. (2006) Participatory mapping and Geographic Information Systems: Whose map? Who is empowered and who disempowered? Who gains and who loses? Electronic Journal of Information Systems in Developing Countries, 25: pp. 1-11.

Coast, S. (2010) State of OpenStreetMap keynote address. State of the Map Conference. Girona, Spain. [Online] Available at: http://wiki.openstreetmap.org/wiki/State_ Of_The_Map_2010 (accessed 3 August 2016).

Crampton, J. (2010) Mapping: A Critical Introduction to Cartography and GIS. Chichester: Wiley-Blackwell.

de Certeau, M. (1984) The Practice of Everyday Life. Berkeley, California: University of California Press.

Deleuze, G. (1995) Negotiations: 1972-1990. New York: Columbia University Press.

Deleuze, G. (2004) Desert Islands and Other Texts (1953-1974). Los Angeles: Semiotext(e).

DeLillo, D. (2010) Point Omega. London: Picador.

Elwood, S. and Leszczynski, A. (2013) New spatial media, new knowledge politics. Transactions of the Institute of British Geographers, 38: pp. 544-559.

Gerlach, J. (2014) Lines, contours, legends: Coordinates for vernacular mapping. Progress in Human Geography, 38: pp. 22-39.

Grosz, E. (2005) Bergson, Deleuze and the becoming of unbecoming. Parallax, 11: pp. 4-13.

Hennion, A. (2007) Those things that hold us together: Taste and sociology. Cultural Sociology, 1: pp. 97-114.

Jackson, J. (1978) Transverse Mercator projection. Survey Review. 24: pp. 278-285.

James, W. (2003) Essays in Radical Empiricism. New York: Dover.

Jellis, T. (2015) Spatial experiments: Art, geography, pedagogy. Cultural geographies in practice. Cultural Geographies, 22: pp. 369-374.

Kitchin, R. and Dodge, M. (2007) Re-thinking maps. Progress in Human Geography, 31: pp. 1-14.

Lacoste, Y. (1973) An illustration of geographical warfare. Antipode, 5: pp. 1-13.

Latour, B. (1993) We Have Never Been Modern. Cambridge, Massachusetts: Harvard University Press.

Latour, B. (2008) Review essay: The Netz-Works of Greek deductions. Social Studies of Science, 38: pp. 441-459.

Leszczynski, A. (2014) On the neo in neogeography. Annals of the Association of American Geographers, 104: pp. 60-79.

Lorimer, H. and Wylie, J. (2010) LOOP (a geography). Performance Research: A Journal of the Performing Arts, 15: pp. 6-13.

McCormack, D. (2014) Atmospheric things and circumstantial excursions. Cultural Geographies, 21: pp. 605-625. 
Newling, J. (2005) Writings by John Newling 1995-2005. London: SWPA.

Orwell, G. (1938) Homage to Catalonia. London: Penguin.

Perkins, C. (2009) 'Playing with maps'. In: Dodge, M., Kitchin, R. and Perkins, C. (eds) Rethinking Maps. London: Routledge, pp. 167-188.

Perkins, C. (2014) Plotting practices and politics: (Im)mutable narratives in OpenStreetMap. Transactions of the Institute of British Geographers, 39: pp. 304-317.

Perkins, C. and Dodge, M. (2008) The potential of user generated cartography: A case study of the OpenStreetMap project and Mapchester mapping party. North West Geography, 8: pp. 19-32.

Rorty, R. (2009) Philosophy and the Mirror of Nature. Princeton, New Jersey: Princeton University Press.

Schaffer, S. (1988) Astronomers mark time: Discipline and the personal equation. Science in Context, 2: pp. 115-145.

Stengers, I. (2005) Introductory notes on an ecology of practices. Cultural Studies Review, 11: pp. 183-196.

Thrift, N. (2012) The insubstantial pageant: Producing an untoward land. Cultural Geographies, 19: pp. 141-168.

Ulfarsson, G. and Unger, E. (2011) Impacts and responses of Icelandic aviation to the 2010 Eyjafjallajökull volcanic eruption: Case study. Transportation Research Record: Journal of the Transportation Research Board, 2214: pp. 144-151.

Whatmore, S. (2002) Hybrid Geographies: Natures, Cultures, Spaces. London: Routledge.

Wylie, J. (2002) An essay on ascending Glastonbury Tor. Geoforum, 33: pp. 441-454.

Zook, M., Dodge, M., Aoyama, Y. and Townsend, A. (2004) 'New digital geographies: Information, communication, and place'. In: Brunn, S., Cutter, S., Harrington, J. (eds) Geography and Technology. Dordrecht: Kluwer Academic Publishers, pp. 155-176.

Zook, M. and Graham, M. (2007) The creative reconstruction of the internet: Google and the privatization of cyberspace and DigiPlace. Geoforum, 38: pp. 1322-1343. 